\title{
Modeling of the solar modulation of Jovian electrons in the inner heliosphere
}

\section{R.R. Nndanganeni ${ }^{1}$}

Centre for Space Research, North-West University, Potchefstroom, South Africa

E-mail:20884648@nwu.ac.za

\section{M.S. Potgieter}

Centre for Space Research, North-West University, Potchefstroom, South Africa

E-mail: Marius.Potgieter@nwu.ac.za

\begin{abstract}
The challenge regarding the modeling of the solar modulation of Jovian electrons lies in determining a reasonable source function which on its part influences the energy range where these particles dominate in the heliosphere. Another controversial issue is what the spectral index of these electrons should be, from the lowest to the highest energies of relevance to solar modulation. If this can be reasonably determined, it becomes possible to compute what the intensity of galactic electrons is at these rather low energies at the Earth. In this study the spectral shape and the value of the Jovian electron source function is computed, using a 3D numerical modulation model. Comparing the modeling with observations, a new source function is determined, and when used in the model, indicates that Jovian electrons can dominate electron intensities in the inner heliosphere only up to about $25 \mathrm{MeV}$ above which the contribution from galactic electrons becomes progressively larger.
\end{abstract}

The 34th International Cosmic Ray Conference

30 July- 6 August, 2015

The Hague, The Netherlands

\section{${ }^{1}$ Speaker}




\section{Introduction}

It is well established that Jupiter's magnetosphere is an important and relatively continuous source of relativistic electrons in interplanetary space [1]. Jovian electrons are emitted from Jupiter's magnetosphere and are observed from inside the orbit of the Earth to a distance as far as $40 \mathrm{AU}$ in the inner heliosphere. They effectively dominate the low energy electron spectrum within the first $\sim 20 \mathrm{AU}$ from the Sun. A controversial issue is what the spectral index of Jovian electrons should be, from the lowest to the highest energies of relevance to solar modulation. It is well-known that the source function can be influenced by solar events such as CIRs and solar flares but most electron observations were reported for solar quite times, so that it is unlikely that the overall spectral index will change significantly as these particles are transported from Jupiter to the point of observation at or close to the Earth.

According to the literature, the Jovian electron source function may have a spectral index ranging from $\gamma=-1.5$ to $\gamma=-3.0$, based mostly on observations away from Jupiter. The spectral shape and the value of the Jovian source function is therefore investigated using observations and a three dimensional (3D) modulation model. The controversy lies in the spectral index of $\gamma=-1.5$. The question arises about how is it possible for the Jovian magnetosphere to produce such a flat source spectrum if known much stronger sources cannot? Based on observations done at Jupiter but mostly close to and at Earth, source functions for Jovian electrons were constructed in the past which could reproduce some observations. It seems that some of these reports, as well as the observations, were somewhat controversial [compare 5 and 7] so that consensus about how a Jovian electron source function (the intensity when these electrons had already escaped the Jovian magnetosphere) exactly looks like, still appears elusive. This aspect is revisited in this work.

\section{Numerical model}

A full three-dimensional (3D) steady-state numerical model, is used to study the transport and modulation of Jovian and galactic electrons. It includes a Jovian electron source function, gradient, curvature and current sheet drifts and a heliosheath, The relevant modulation processes are combined in Parker's transport equation (TPE) given by:

$$
\frac{\partial f}{\partial t}=-\left(\mathbf{V}+\left\langle\mathbf{v}_{D}\right\rangle\right) \cdot \nabla f+\nabla\left(\mathbf{K}_{S} \cdot \nabla f\right)+\frac{1}{3}(\nabla \cdot \mathbf{V}) \frac{\partial f}{\partial \ln P}+Q,
$$

where $f(\mathbf{r}, P, t)$ is the omni-directional cosmic ray distribution function dependent on position $\mathbf{r}$, rigidity $P$ and time $t$. Here, $\mathbf{V}$ is the solar wind velocity, and $\mathbf{K}_{s}$ is the $3 \mathrm{D}$ diffusion tensor. It is assumed that $\partial f / \partial t=0$ which means the contribution of short-term modulation effects is neglected, with the focus instead on the global spatial and spectral trends. Terms on the right hand side respectively represent convection, gradient and curvature drifts, diffusion and adiabatic energy changes with $Q$ the Jovian electron source function.

This TPE is solved numerical using a 3D model similar to that described in detail by Ferreira [4]. The model and the diffusion coefficients used here are similar to those in [3] and are not repeated here. For details, see also Nndanganeni and Potgieter, this volume. The main aspect 
concerning the diffusion coefficients for electrons is that they are independent of energy below $\sim 100 \mathrm{MeV}$ [3]. A new very local interstellar spectrum is utilized [19, 20, 21].

\section{Results and discussion}

Simnett and McDonald [7], motivated by IMP IV electron observations, reported a spectral index of $\gamma=-1.8$ for the energy range 3-20 MeV. They, however, seemed to have believed that these electrons mostly had a galactic origin which is unlikely. Moses [5] reported $\gamma=-1.5$ while Ferrando et al. [8] reported $\gamma=-2.5$ at these low energies. Recently, Kühl et al. [9], using electron observations near Earth from SOHO/EPHIN, reported even lower values but mainly consistent to what [5] reported and as such examples of how controversial these observations and their interpretation can be. Lopate [6], on his part motivated by the Pioneer 10 observations over two decades, calculated $\gamma=-(1.8 \pm 0.2)$ in the energy range $2 \mathrm{MeV} \leq E \geq 17 \mathrm{MeV}$ as shown in his Figure 3. He found that it changed from $\gamma=-1.2$, close to Earth in 1973, to smaller values from 1975 onwards. However, Lopate's [6] result is close to $\gamma \sim 2$ as given by [10]. Others $[11,12,13]$, making use of SOHO/EPHIN observations, claimed that the electrons in the energy range of $150 \mathrm{keV}$ to $10 \mathrm{MeV}$ observed during the quite time period in 1996 were indeed of Jovian origin with a spectral index ranging from $\gamma=-1.5$ to $\gamma=-1.7$, consistent to what [6] and others reported for observations closer to the Earth.

For this modeling study, two Jovian electron source functions are investigated. A previously used source function for electrons at Jupiter is taken from [4] and is given by

$$
\begin{aligned}
& Q=j_{\text {source }}=1.5\left(\frac{c_{k} j_{1.5} d_{k} j_{6.0}}{c_{k} j_{1.5}+d_{k} j_{6.0}}\right) \\
& j_{1.5}=5.0 \times 10^{3}\left(E / E_{0}\right)^{-1.5}, j_{6.0}=1.0 \times 10^{9}\left(E / E_{0}\right)^{-6.0} ; c_{k}=0.6 ; d_{k}=5.0 .
\end{aligned}
$$

This function, with differential intensity $\mathrm{j}\left(\mathrm{m}^{-2} \mathrm{~s}^{-1} \mathrm{sr}^{-1} \mathrm{MeV}^{-1}\right)$ and kinetic energy $\mathrm{E}$ (in $\mathrm{GeV}$ ), with $\mathrm{E}_{0}=1 \mathrm{GeV}$, is a combination of two power-laws, $j \propto E^{-1.5}$ and $j \propto E^{-6.0}$ and was constructed to be compatible with normalized ISEE 3 (ICE) spectra [5] and Pioneer 10 data [6] when Pioneer 10 was in or close to the Jovian magnetosphere.

This source function serves as a reference for the work to follow. Inspection of this source function indicates that it is not steep enough to explain recent Jovian electron observations and needs to be re-evaluateded. Revisiting the possible shape and intensity value of the Jovian electron source function an alternative source function is constructed for this work and the results are compared to that of [4] in terms of computed modulated spectra at the Earth (1 AU). 
This source function is again a combination of two power-laws $j(E) \propto E^{-3.0}$ and $j \propto E^{-12.0}$ which are both much steeper than the one given above.

It is:

$$
\begin{aligned}
& Q=j_{\text {source }}=30\left(\frac{c_{k} j_{3.0} d_{k} j_{12.0}}{c_{k} j_{3.0}+d_{k} j_{12.0}}\right) \\
& j_{3.0}=1.5 \times 10^{3}\left(E / E_{0}\right)^{-3.0}, j_{12.0}=1.0 \times 10^{16}\left(E / E_{0}\right)^{-12} ; c_{k}=d_{k}=5.0 .
\end{aligned}
$$

Modeling results based on the source functions as given by Eq. (2) and Eq. (3) are shown in Figure 1. It shows the comparison between the two source functions together with their modulated spectra at Earth. This alternative source function differs in terms of how its differential intensity below $10 \mathrm{MeV}$ scales as function of energy; as indicted above, it scales as $j(E) \propto E^{-3.0}$ below $10 \mathrm{MeV}$, while softening significantly to $j \propto E^{-12.0}$ above this energy.

This is more consistent with an exponential cut-off of these accelerated electrons, instead of a power-law as used by [4]. The observations are from ISEE 3 (ICE) from 1979 to 1984 as reported by Moses [5, see his Figure 13]. The differences illustrate the implication of using a much steeper source function at all energies. Later observations (Webber, private communication, 2014) from when Voyager 1 was close to Jupiter, is closer to this source function but since the data are not yet published these observations could not be used here. Including a more comprehensive set of observations will be the next step [16; see also 20].

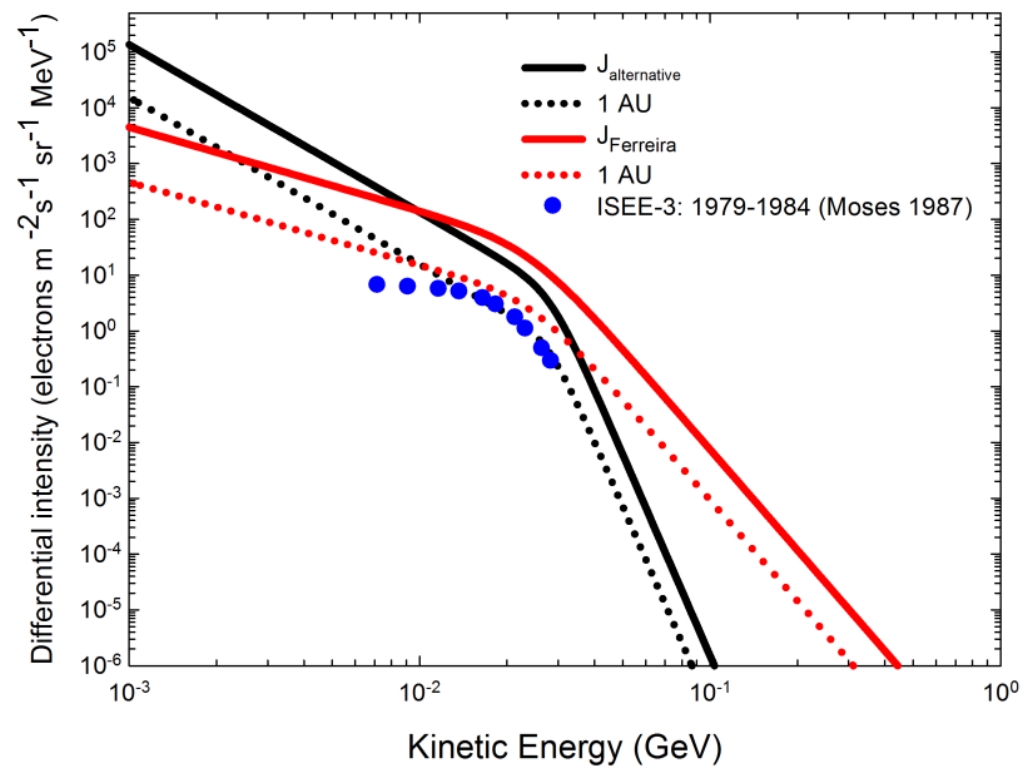

Figure 1: A comparion of the new alternative source function (Eq. 3; black solid line) for Jovian electrons with the source function (Eq. 2; red solid line) of [4]. The dotted lines are the corresponding computed, modulated spectra at Earth (1 AU). The observations are from Moses [5]. 
Qualitatively, the source function in Eq. (2), is interpreted as underestimating the Jovian electron intensity at lower energies $(E<\sim 10 \mathrm{MeV})$ but significantly over-predicting the intensity at higher energies so that in his case the Jovian electrons would dominate up to much higher energies than what the computed spectrum at Earth is suggesting, based on Eq. (3) as an alternative source function. However, because of the complexity of low-energy electron observations and modeling, and taking into account all the solar and other phenomena that may influence these observations, it is conceded that there is not a conclusive reason to say that Eq. (2) is incorrect. It is argued that the new alternative source function is preferred. Of course, it could be that the shape of the Jovian source function is changed by propagation conditions towards the Earth and that the assumption that the diffusion coefficients for electrons are independent of energy [3] at these low energies could be an oversimplification. However, the theory (see e.g. [14]) seems rather solid about this so if this assumption is valid it may mean that the flatness of the observed spectra close to Earth is caused by the contribution of galactic electrons at these energies. This aspect seems to have been neglected up to now [e.g. 9,12,17,23]. The new source function (Eq. 3) allows that galactic electrons above $10-20 \mathrm{MeV}$ are making a larger contribution to the total electron intensity in the innermost heliosphere than previously thought (see also $[18,22]$.

\section{Conclusions}

The issue under debate here is what the spectral index of Jovian electrons should be. According to the literature, the Jovian electron source function may have a spectral index ranging from $\gamma=-1.5$ to $\gamma=-3.0$, based mostly on observations away from Jupiter $[6,8,15]$. Theoretical arguments, however, preclude an electron accelerated spectrum to have $\gamma>-2$. The spectral shape and value of the Jovian source function is revisited and is computed using a 3D modulation model in comparison with observations. A new alternative source function is constructed as shown in Figure 1, with a spectral index that scales as in Eq. (3). The differences between the considered two source functions illustrate the controversy, and additionally the implication of using a much steeper source function at all energies. It may mean that the flatness of the observed spectra close to Earth is caused by the contribution of galactic electrons. This aspect seems to have been ignored up to now. For the new source function the implication is that Jovian electrons could dominate up to only $25 \mathrm{MeV}$. 


\section{Acknowledgements}

MSP expresses his gratitude for the partial funding by the South African National Research Foundation (NRF). RRN thanks the NRF and the South African Space Agency (SANSA) for partial financial support during her $\mathrm{PhD}$-study.

\section{References}

[1] Simpson, J. A., Hamilton, D., Lentz, G., et al., Science 183, 306, 1974.

[2] Ferreira, S. E. S., Potgieter, M. S., et al., J. Geophys. Res. 106, 24979, 2001.

[3] Potgieter, M. S., and Nndanganeni, R. R., Astrophys. Space Sci. 345, 33, 2013.

[4] Ferreira, S. E. S., Ph.D. thesis, Potchefstroom University, South Africa, 2002.

[5] Moses, D., Astrophys. J. 313, 471, 1987.

[6] Lopate, C., Proc. $22^{\text {nd }}$ Int. Cosmic Ray Conf. 2,149, 1991.

[7] Simnett, G. M., and McDonald, F. B., Astrophys. J. 157, 1435, 1969.

[8] Ferrando, P., Müller-Mellin, R., et al., Proc. $22^{\text {nd }}$ Inter. Cosmic Ray Conf. 3, 366, 1991.

[9] Kühl, P., Dresing, N., et al., Proc. $33^{\text {rd }}$ Inter. Cosmic ray Conf., 2013.

[10] Chenette, D. L., J. Geophys. Res. 85, 2243, 1980.

[11] Gómez-Herrero, R., Rodríguez-Frías, M. D., et al., Solar Phys. 194, 405, 2000.

[12] Gómez-Herrero, R., et al., Proc. Inter. Cosmic Ray Conf. 36012001.

[13] Rodríguez, M. D., Gómez-Herrero, R., et al., Proc. $1^{\text {st }}$ ESA SP 463, 415, 2000.

[14] Potgieter, M. S., J. Geophys. Res. 101, A11, 24411, 1996.

[15] Eraker, J. H., Astrophys. J. 257, 862, 1982.

[16] Nndanganeni, R.R., Ph.D. thesis, North-West University, South Africa, in preparation.

[17] Strauss, R. T., Potgieter, M. S., Ferreira, S. E. S., Adv. Space Res. 51, 339, 2013.

[18] Potgieter, M. S., Webber, W. R., Boezio, M., et al., in preparation.

[19] Potgieter, M. S., Vos, E. E., Nndanganeni, R. R., Proc. 14th ICATPP Conference, 8, 1, 2014.

[20] Potgieter, M. S., Nndanganeni, R. R., Vos, E. E., Boezio, M., Proc. $33^{\text {rd }}$ Inter. Cosmic Ray Conf., 2013.

[21] Potgieter, M.S., Nndanganeni, R. R., Vos, E. E., Boezio, Munini, R., Proc. $33^{\text {rd }}$ Inter. Cosmic Ray Conf., 2013.

[22] Nndanganeni, R. R., Potgieter, M. S., Proc. 33 ${ }^{\text {rd }}$ Inter. Cosmic Ray Conf., 2013. 
Modeling of the solar modulation of Jovian electrons

[23] Dunzlaff, P., Heber, B., Kopp, A., Potgieter, M. S., Proc. $33^{\text {rd }}$ Inter. Cosmic Ray Conf., 2013. 